\title{
Factorization of linear partial differential operators and Darboux integrability of nonlinear PDEs* (ISSAC'98 Poster)
}

\author{
S.P.Tsarev \\ Department of Mathematics \\ Krasnoyarsk State Pedagogical University \\ Lebedevoi, 89 \\ 660049, Krasnoyarsk, Russia \\ e-mail: tsarev@edk.krasnoyarsk.su
}

\begin{abstract}
Using a new definition of generalized divisors we prove that the lattice of such divisors for a given linear partial differential operator is modular and obtain analogues of the well-known theorems of the Loewy-Ore theory of factorization of linear ordinary differential operators. Possible applications to factorized Gröbner bases computations in the commutative and non-commutative cases are discussed, an application to finding criterions of Darboux integrability of nonlinear PDEs is given.
\end{abstract}

\section{Introduction}

Factorization is often used for simplification of solution procedures for polynomials (factorized Gröbner bases computations) and linear ordinary differential operators (LODO). It is well-known that every (multivariate) polynomial factors into product of irreducible polynomials (in the given coefficient field) in a unique way; for LODO an analogous result had been proved by E.Landau [15] and in a more precise form by A.Loewy [16, 17]: any two different decompositions of a given LODO $L$ into products of irreducible LODO $L=P_{1} \circ \cdots \circ P_{k}=\bar{P}_{1} \circ \cdots \circ \bar{P}_{p}$ have the same number of factors $(k=p)$ and the factors are pairwise similar (in some transposed order). Two (irreducible for simplicity) LODO $L$ and $M$ are called similar (or operators of the same type) if one can find operators $A$ and $B$ such that $\operatorname{ord}(A)=\operatorname{ord}(B)<\operatorname{ord}(L)=\operatorname{ord}(M)$ and $A \circ L=M \circ B$ (see below for more detail). The theory of factorization of LODO (Loewy-Ore theory) was developed in [16, 17, 19]. From the algorithmic point of view factorization of LODO was addressed for the first time in [2] where an outline of an algorithm for factorization of LODO with coefficients in the simplest differential field of rational functions (i.e. over $\overline{\mathbf{Q}}(x))$ was given. In the past decade many improvements of this algorithm and alternative algorithms were proposed (see

\footnotetext{
${ }^{*}$ The research described in this article was partially supported by INTAS grant 95-IN-RU-412 and Russian Presidential grant 9615-96834
}

ISSAC'98 Poster, 13-15 August 1998, Rostock, Germany
27] and references therein), applications to the differential Galois group computation were given in [23].

Unfortunately very little is known about factorization properties of linear partial differential operators (LPDO). The following interesting example was given by E.Landau (see [5]): if

$$
\begin{gathered}
P=D_{x}+x D_{y}, \quad Q=D_{x}+1, \\
R=D_{x}^{2}+x D_{x} D_{y}+D_{x}+(2+x) D_{y},
\end{gathered}
$$

then $L=Q \circ Q \circ P=R \circ Q$. On the other hand the operator $R$ is absolutely irreducible, i.e. one can not factor it into product of first-order operators with coefficients in any extension of $\mathbf{Q}(x, y)$.

This example shows that in order to develop a "good" theory of factorization of LPDO one shall try to use some generalization of the notion of a factor (divisor) for LPDO. Such tricks are very common in the commutative case, as the first example we may cite Kummer-Dedekind theory of divisors for algebraic number rings. As proposed by Dedekind we may use ideals of the ring of algebraic integers of a given (finite) extension of $\mathbf{Q}$. Since not all ideals in this ring are principal ideals (i.e. they are not generated as multiples of a single element) we obtain an extension of the notion of a divisor and this suffices (cf. for example [10, Ch.12]) to obtain uniqueness of decomposition of any algebraic integer (i.e. of the principal ideal it generates) into product of prime "ideal" divisors.

For the non-commutative ring of LODO (with coefficients in some differential field, for simplicity we will suppose that coefficients belong to $\overline{\mathbf{Q}}(x)$ i.e. they are rational functions with arbitrary algebraic number coefficients) one can use the Euclid division algorithm to prove that any left or right ideal in this LODO ring $\mathbf{Q}(x)\left[D_{x}\right]$ is a principal ideal; there are no nontrivial two-sided ideals. So there is no possibility (and necessity) of "ideal" generalization of the notion of divisors, the only implication of non-commutativity results in "similarity" of factors in different factorizations of a given LODO.

Another well-known "unique factorization" theorem is the classical Jordan-Hölder theorem in the theory of finite groups (or finitely generated modules).

In the first half of the XX century a common approach to these (and many more) cases was proposed. Let us introduce the obvious partial order in the set of (left) ideals: $I_{1} \leq I_{2}$ if $I_{1} \supset I_{2}$; we call $I_{1}$ a divisor of $I_{2}$ in such a case. Then instead of factorizations

$$
L=L_{1} \circ \ldots \circ L_{k}
$$


of an element $L$ of the ring we will consider chains $|L\rangle>$ $\left|L_{2} \circ \ldots \circ L_{k}\right\rangle>\left|L_{3} \circ \ldots \circ L_{k}\right\rangle>\ldots>\left|L_{k}\right\rangle>0=|1\rangle$ of corresponding (left) principal ideals. Irreducibility of factors corresponds to maximality of this chain, i.e. impossibility to insert intermediate ideals between any two elements of the chain. The partially ordered set $\mathcal{M}$ (also called poset) of ideals in the cited above "good" cases has the following two fundamental properties:

a) for any two elements $A, B \in \mathcal{M}$ one can find a unique $C=\sup (A, B)$, i.e. such $C$ that $C \geq A, C \geq B$, and $\forall X \in$ $\mathcal{M},(X \geq A, X \geq B) \Rightarrow X \geq C$. Analogously there exist a unique $D=\inf (A, B), D \leq A, D \leq B, \forall X \in \mathcal{M},(X \leq$ $A, X \leq B) \Rightarrow X \leq D$. Such posets are called lattices. $\sup (A, B)$ and $\inf (\bar{A}, B)$ correspond to the least common multiple and the greatest common divisor for the cases of number rings and LODO. In our cases a lattice will always have zero i.e. an element $0 \in \mathcal{M}$ such that $\forall X \in \mathcal{M}, X \geq$ 0 . For simplicity (and following the established tradition) $\sup (A, B)$ will be hereafter denoted as $A+B$ and $\inf (A, B)$ - as $A \cdot B$;

b) For any three $A, B, C \in \mathcal{M}$ the following modular identity holds:

$$
(A \cdot C+B) \cdot C=A \cdot C+B \cdot C
$$

This weaker form of distributivity was discovered by Dedekind. The theory of modular lattices (i.e. posets with the above two properties, such posets are also called "Dedekind structures") has beautiful (for our purpose :-) results. Namely these two simple properties are sufficient to prove the following four elegant theorems (cf. [3], [, 11]):

Theorem 1 (Jordan-Hölder-Dedekind chain condition) Any two finite maximal chains

$$
\begin{gathered}
L>L_{1}>\cdots>L_{k}>0 \\
L>M_{1}>\cdots>M_{r}>0
\end{gathered}
$$

for a given $L \in \mathcal{M}$ have equal length: $k=r$.

Theorem 2 (Kurosh \& Ore) If $L=L_{1}+L_{2}+\ldots+L_{p}=$ $M_{1}+M_{2}+\ldots+M_{r}$ are two noncancellable sup-representations of $L \in \mathcal{M}$ then $p=r$ and for every $L_{i}$ one can find $M_{j}$ such that $L=L_{1}+\ldots+L_{i-1}+M_{j}+L_{i+1}+\ldots+L_{p}$.

We recall that a sup-representation

$$
L=L_{1}+L_{2}+\ldots+L_{k}
$$

is called non-cancellable if $\forall i,\left(L_{1}+\ldots+L_{i-1}+L_{i+1}+\right.$ $\left.\ldots+L_{k}\right) \neq L$ and each $L_{i}$ can not be sup-represented: $L_{i} \neq$ $A_{i}+B_{i}$ for $A_{i} \neq L_{i}, B_{i} \neq L_{i}$.

We call a sup-representation (B) a direct sum if the set $\left\{L_{i}\right\}$ is independent that is $\forall i,\left(L_{1}+\ldots+L_{i-1}+L_{i+1}+\right.$ $\left.\ldots+L_{k}\right) \cdot L_{i}=0$ and each $L_{i}$ can not be represented as a sum of two independent elements. Direct sums are denoted $L=L_{1} \oplus L_{2} \oplus \ldots \oplus L_{k}$. An element $A \in \mathcal{M}$ is called indecomposable if $A \neq B \oplus C, B \neq 0, C \neq 0$.

Theorem 3 (O.Ore) Let an element $L$ of a modular lattice have finite maximal chains (A) and $L=L_{1} \oplus \ldots \oplus L_{k}=$ $M_{1} \oplus \ldots \oplus M_{r}$ with indecomposable $L_{i}, M_{j}$. Then $k=r$ and $\forall L_{i}$ one can find $M_{j}$ such that $L=L_{1} \oplus \ldots \oplus L_{i-1} \oplus M_{j} \oplus$ $L_{i+1} \oplus \ldots \oplus L_{p}$.

Let us call $l(L):=k+1$ the length of $L \in \mathcal{M}$ if $L$ has a finite maximal chain (4) (of length $k+1$ ). We set $l(0)=0$. The length of a LODO is equal to the number of irreducible factors in decomposition (2).
Theorem 4 If all elements of a modular $\mathcal{M}$ have finite length then $l(A+B)+l(A \cdot B)=l(A)+l(B)$.

These theorems give a unified approach to many well known facts in the theory of groups (and group representations), commutative and non-commutative rings; in particular they encompass many results of the Loewy-Ore theory of factorization of LODO.

Let us prove here for completeness that the poset of (left) ideals of a (non-commutative) ring is a modular lattice. Firstly we notice that $\sup (A, B)=A+B$ corresponds to the intersection of ideals $A, B$; $\inf (A, B)=A \cdot B$ corresponds to the ideal composed of the sums $a+b, a \in A$, $b \in B$. Then if $x \in A \cdot C+B \cdot C$ (the r.h.s of (3)) then $x \in C, x=a+b, a \in A, a \in C, b \in B, b \in C$. Obviously $a+b \in C, a+b \in A \cdot C+B$, so $A \cdot C+B \cdot C \subset(A \cdot C+B) \cdot C$. Vice versa if $x \in(A \cdot C+B) \cdot C$ then $x \in C, x=a+b$, $a \in A, a \in C, b \in B \Rightarrow b=x-a \in C$ so $b \in B \cdot C$ and $x \in A \cdot C+B \cdot C$ which proves $(A \cdot C+B) \cdot C \subset A \cdot C+B \cdot C$.

The basic notion of similarity also exists for modular lattices:

Definition 1 Two elements $A, B$ of a modular lattice $\mathcal{M}$ are called similar if one can find $C \in \mathcal{M}$ such that $A \cdot C=$ $B \cdot C=0$ and $A+C=B+C$ (i.e. $A \oplus C=B \oplus C$ ).

We will need also the notion of similarity of intervals or quotients $[B / A]:=\{X \in \mathcal{M} \mid A \leq X \leq B\}$ for pairs $A \leq B$.

Proposition 1 If $A, B$ are elements of a modular lattice $\mathcal{M}$ then the intervals $I_{1}=[A /(A \cdot B)]$ and $I_{2}=[(A+B) / B]$ are projective, $I_{1} \sim I_{2}$, i.e. isomorphic with specific poset isomorphisms $\phi: I_{1} \rightarrow I_{2}, \phi(X)=X+B, \psi(X)=A \cdot X$, $\psi=\phi^{-1}$.

Definition 2 Two intervals $\left[B_{1} / A_{1}\right],\left[B_{2} / A_{2}\right]$ are called similar if there exists a finite sequence of projective intervals $\left[B_{1} / A_{1}\right] \sim I_{1} \sim I_{2} \sim \ldots \sim I_{k} \sim\left[B_{2} / A_{2}\right]$.

One can prove that in Theorems 13 the corresponding factors (intervals) are similar (in some transposed order). Similarity of intervals in Theorem 1 gives similarity of the respective irreducible factors in (2) or isomorphism of the factorgroups (factormodules) for the modular lattice of normal subgroups of a given (finite) group (resp. submodules).

The case of the ring of LPDO is more complicated. It has no two-sided ideals and left (right) ideals are no longer principal ideals in the general case. Certainly the poset of all left (right) ideals is a modular lattice. But unfortunately we can not use the above results: for a LPDO $L$ we get finite chains,(4) of left ideals (the ring of LPDO is Noetherian, see [4]) but the intervals in any chain are not (as a rule) "irreducible" i.e. one can always insert intermediate ideals between some of them so the length of chains (44) for a given $L$ is not bounded. For example for arbitrary LODO $L \in \mathbf{Q}(x)\left[D_{x}\right] \subset \mathbf{Q}(x, y)\left[D_{x}, D_{y}\right]$ we can take $|L\rangle>|L\rangle+\left|D_{y}^{m}\right\rangle>|L\rangle+\left|D_{y}^{m-1}\right\rangle>\ldots|L\rangle+\left|D_{y}\right\rangle>$ $0=\mathbf{Q}(x, y)\left[D_{x}, D_{y}\right]$. Even the simplest $D_{x}$ becomes "reducible"! Similar infinite examples exist for decompositions into (direct) sup-sums. So Theorems 14 are useless.

We conclude that the poset of all (left) ideals of LPDO is too "large". For the commutative case of multivariate polynomials one can limit oneself to principal ideals and get the desired modular lattice with finite chains. Again for LPDO the poset of (left) principal ideals is too "small": it does not form even a lattice. For example for the two operators $P, Q$ in (1) the intersection of the left principal ideals $|P\rangle \bigcap|Q\rangle$ 
(their "LCM") is no longer principal: one can easily check directly that there are no second-order common left multiples of both $P, Q$ but we have two linearly independent third-order operators divisible by $P, Q$ :

$$
\begin{gathered}
L_{31}=\left(x D_{x} D_{y}+(x-1) D_{y}-D_{x}-1\right) \circ P= \\
\left(x^{2} D_{y}^{2}+x D_{x} D_{y}-(x+1) D_{y}-D_{x}\right) \circ Q, \\
L_{32}=\left(D_{x}^{2}+2 D_{x}+1\right) \circ P=Q \circ Q \circ P= \\
\left(D_{x}^{2}+x D_{x} D_{y}+(x+2) D_{y}+D_{x}\right) \circ Q=R \circ Q,
\end{gathered}
$$

so there is no "least" common left multiple. Analogously we can directly check that these $L_{31}, L_{32}$ have only $Q, P$ as their common right divisors so $L_{31}, L_{32}$ have no "greatest" right common divisor. Also as the E.Landau's example shows the Jordan-Hölder-Dedekind chain condition fails for principal ideals.

Below (section 2) we define an "intermediate" poset of "codimension 1" left ideals which is larger than the poset of principal left ideals but smaller than the lattice of all left ideals. This new poset of "generalized divisors" provides all the necessary properties: it is a modular lattice with finite maximal chains (4) for every element and finite decompositions into (direct) sup-sums so the basic Theorems 14 are applicable; any first-order LPDO is irreducible and any LODO $L$ irreducible in $\mathbf{Q}(x)\left[D_{x}\right]$ remains irreducible (as LPDO) in our poset. This is our main result.

For applications the most important property of our modular lattice of generalized divisors would be certainly the possibility to decompose operators into sup-sums in an overdetermined system of LPDO

$$
\left\{\begin{array}{c}
L_{1} f=0, \\
L_{2} f=0, \\
\cdots \\
L_{k} f=0 .
\end{array}\right.
$$

Suppose that $L_{1}=A_{1}+\ldots A_{p}$ for some left ideal divisors then since each $A_{i}$ is finitely generated (see [4]): $A_{i}=$ $\left|L_{i 1}, \ldots, L_{i s_{i}}\right\rangle$, we can decompose (6) into union of systems

$$
\left\{\begin{array} { c } 
{ L _ { 1 1 } f = 0 , } \\
{ \cdots } \\
{ L _ { 1 s _ { 1 } } f = 0 , } \\
{ L _ { 2 } f = 0 , } \\
{ \cdots } \\
{ L _ { k } f = 0 . }
\end{array} \quad \cdots \quad \left\{\begin{array}{c}
L_{p 1} f=0, \\
\cdots \\
L_{p s_{p}} f=0, \\
L_{2} f=0, \\
\cdots \\
L_{k} f=0 .
\end{array}\right.\right.
$$

Sums of solutions of (⿻) are obviously solutions of (6) and we conjecture that they span the whole space of solutions of (6). Also we need an algorithm for such sup-decompositions of LPDO (see section 5 for the discussion).

Substitution of (6) with (7) is an analogue of the wellknown factorization technique for commutative Gröbner bases computations. This technique considerably reduces the complexity of computations in many practical cases. The overdetermined systems of type (6) with one or many unknown functions are typical in many applications ( 18, 25): computation of conservation laws, symmetries and invariant solutions of systems and single nonlinear ODEs and PDEs. For any system (6) one may use the standard Janet-Riquier technique ([13, 21, 22, 25]) of reduction of (6) to the so called passive (standard, normal) form. In the case of constant coefficient systems (6) (in the commutative case) this algorithm practically coincides with the Gröbner algorithm (for total degree+weight ordering). Unfortunately the complexity of Janet-Riquier algorithm is very high even for modest LPDO systems. Recently one interesting contribution to reduction of the complexity for computation of the genus (roughly speaking this is the "dimension" of the solution space) of (6) was given in 9. Our approach may help in decomposition of the solution space of (6) into "irreducible submanifolds". Further possible generalizations and applications to the commutative case are discussed in section .

Another connection of our definition of factorization of LPDO and integrability properties of nonlinear PDEs is discussed in section 4 the established in 11, 14, 26] criterion of Darboux integrability [6] ("explicit" integrability) of such nonlinear PDEs is equivalent to generalized factorization of the corresponding linearized equation. This gives a new insight into possible generalizations of the notion of Darboux integrability of higher-order nonlinear PDEs which is now under investigation.

\section{Divisor ideals of LPDO}

We study general LPDO

$$
L=\sum_{|\vec{i}| \leq m} a_{i_{1} \cdots i_{n}}(\vec{x}) D_{x_{1}}^{i_{1}} D_{x_{2}}^{i_{2}} \cdots D_{x_{n}}^{i_{n}},
$$

$|\vec{i}|=i_{1}+\ldots+i_{n}, \vec{x}=\left(x_{1}, \ldots, x_{n}\right), D_{x_{i}}=\partial / \partial x_{i}, \operatorname{ord}(L):=$ $m$. For simplicity and without loss of generality we will suppose that the number of the independent variables is $n=2, x:=x_{1}, y:=x_{2}$, and the coefficients $a_{i j}(x, y)$ in (8) are rational functions with rational coefficients, $a_{i j}(x, y) \in$ $\mathbf{Q}(x, y)$, so $L \in \mathbf{Q}(x, y)\left[D_{x}, D_{y}\right]$.

It is straightforward to check that for every finite set of LPDO $L_{1}, \ldots, L_{k}$ one may algorithmically find all their common left multiples (left c.m.) up to fixed order $N$ : take $M_{1} \circ L_{1}=\ldots=M_{k} \circ L_{k}$ with $\operatorname{ord}\left(M_{i}\right)=N-\operatorname{ord}\left(L_{i}\right)$ and indefinite coefficients, then we get a linear algebraic (not differential!) system for the coefficients of $M_{i}$; the number of equations in this linear system will be less than the number of the unknown coefficients for sufficiently large $N$, so the set of left (right) c.m. is always nonempty.

All these and subsequent results are certainly invariant w.r.t. substitution of left ideal with right ideals; application of the usual adjoint operation will suffice for this purpose. We will denote the left (right) principal ideal generated by LPDO $L$ with $|L\rangle$ (resp. $\langle L|$ ).

Definition 3 The left LPDO ideal $\operatorname{lLCM}\left(\left|L_{1}\right\rangle, \ldots,\left|L_{k}\right\rangle\right):=$ $\left|L_{1}\right\rangle \bigcap \ldots \bigcap\left|L_{k}\right\rangle$ is called the left least common multiple of $L P D O L_{i}$.

This ILCM is always non-empty and (see Introduction) not principal in the general case.

Definition 4 The same ideal $\left|L_{1}\right\rangle \bigcap \ldots \bigcap\left|L_{k}\right\rangle$ will be also called the left greatest common divisor of $L_{i}$.

Remark. This is serious :-) Below the reader will see that this is the key to the whole trick.

Definition 5 We call two LPDO L, $R$ a (generalized) divisor operator couple for $L P D O M$ if there exist $L P D O X, Y$, $Q$ such that

$$
\begin{gathered}
X \circ M=Y \circ R, \\
X \circ L=Y \circ Q .
\end{gathered}
$$


One may informally think that $M \cong L \circ R$ and if in fact $M$ factors into the product of $L, R$ then we may choose $X=Q=1, Y=L$ in (9).

A divisor operator couple is called nontrivial if $\operatorname{ord}(L)>$ $0, \operatorname{ord}(R)>0$ and $L, R$ are not divisible by $M$, i.e. $L \neq$ $M \circ P, R \neq K \circ M$. In this case we will say that the operators $M$ and $R$ ( $M$ and $L$ ) have nontrivial (generalized) right (resp. left) common divisor.

Remark. These definitions actually say that we can restore the (greatest) common divisor if we can find (least) common multiples; for the case of LODO if $Z=\operatorname{lLCM}(M, R)=$ $X \circ M=Y \circ R$ and $V=\operatorname{rLCM}(X, Y)=X \circ L=Y \circ Q$ then $M=L \circ G, R=Q \circ G, G=\operatorname{rGCD}(M, R)$. This explains Definition 4. For integral domains (commutative rings with 1 and no zero divisors) if two elements have LCM (i.e. some common multiple such that this c.m. divides any other c.m.) then they automatically have GCD (i.e. some common divisor such that this divisor is divided by any other common divisor); the converse is not true in general; also $\operatorname{LCM}(a c, b c)=c \cdot \operatorname{LCM}(a, b)$ when one of them exist, this is again not true for GCD (the author thanks Dr. N.N.Osipov who communicated to him these facts for integral domains).

Lemma 1 If (9) holds and for some $X_{1}, Y_{1}$ we have $X_{1} \circ$ $M=Y_{1} \circ R$, then $X_{1} \circ L=Y_{1} \circ Q$.

Proof. Let us find some left c.m. of $X_{1}, X: \tilde{X}=\bar{X} \circ X_{1}=$ $\bar{X}_{1} \circ X$. Then $\bar{X} \circ X_{1} \circ M=\bar{X} \circ Y_{1} \circ R=\bar{X}_{1} \circ X \circ M=$ $\bar{X}_{1} \circ Y \circ R$ so (since the ring of LPDO has no zero divisors) $\bar{X} \circ Y_{1}=\bar{X}_{1} \circ Y$ and $\bar{X}_{1} \circ Y \circ Q=\bar{X}_{1} \circ X \circ L=\bar{X} \circ X_{1} \circ L=$ $\bar{X} \circ Y_{1} \circ Q$ hence $X_{1} \circ L=Y_{1} \circ Q$.

So (9) does not depend on the choice of the $\operatorname{lLCM}(M, R)=$ $X \circ M=Y \circ R$.

Lemma 2 If (马) holds and some right c.m. of $M, L$ is chosen $M \circ \bar{X}=L \circ \bar{Y}$ then

$$
\begin{aligned}
& M \circ \bar{X}=L \circ \bar{Y}, \\
& R \circ \bar{X}=Q \circ \bar{Y} .
\end{aligned}
$$

Proof. $Y \circ Q \circ \bar{Y}=X \circ L \circ \bar{Y}=X \circ M \circ \bar{X}=Y \circ R \circ \bar{X} \Rightarrow$ $Q \circ \bar{Y}=R \circ \bar{X}$.

From (9) and Lemma 11 we conclude that the set of operators $L$ forming a generalized divisor couples with fixed $R$, $M$ is a right ideal $\{L \mid$; from (10) we see that for fixed $M, L$ operators $R$ form a left ideal $\{R$.

Definition 6 Left ideal $\mid R\}$ and right ideal $\{L \mid$ form a (generalized) divisor ideal couple for an operator $M$ (we denote this fact as $\{L|M| R\})$ if:

a) any $R \in \mid R\}, L \in\{L \mid$ form a divisor operator couple for $M$ i.e. (\$) holds;

b) if some LPDO L forms divisor operator couples for $M$ with every $R \in \mid R\}$ then $L \in\{L \mid$;

c) if some $L P D O R$ forms divisor operator couples for $M$ with every $L \in\{L \mid$ then $R \in \mid R\}$.

Lemma 3 Let $\{L|M| R\}$ be a divisor ideal couple for $M$. Then for every $\left.M_{1} \in \mid R\right\}$ we can find a unique right ideal $\left\{Q_{1} \mid\right.$ such that $\left\{Q_{1}\left|M_{1}\right| R\right\}$.

Proof. Since $\left.M_{1} \in \mid R\right\}$ then for every $L \in\{L \mid$ we have the unique (Lemma 11) $Q_{1}$ such that $\exists X_{1}, Y_{1}$,

$$
\begin{gathered}
X_{1} \circ M=Y_{1} \circ M_{1}, \\
X_{1} \circ L=Y_{1} \circ Q_{1} .
\end{gathered}
$$

Take some left c.m. of $M_{1}, R$ for $\left.R \in \mid R\right\}: Z_{1}=X_{M_{1}} \circ M_{1}=$ $Y_{M_{1}} \circ R$, and some left c.m. of $Y_{1}, X_{M_{1}}: Z_{2}=\bar{Y}_{1} \circ X_{M_{1}}=$ $\bar{X}_{M_{1}} \circ Y_{1}$. Then $\bar{X}_{M_{1}} \circ X_{1} \circ M=\bar{X}_{M_{1}} \circ Y_{1} \circ M_{1}=\bar{Y}_{1} \circ X_{M_{1}} \circ$ $M_{1}=\bar{Y}_{1} \circ Y_{M_{1}} \circ R$, so we get $\bar{X}_{M_{1}} \circ X_{1} \circ M=\bar{Y}_{1} \circ Y_{M_{1}} \circ R$. Using Lemma 11 we conclude $\bar{X}_{M_{1}} \circ X_{1} \circ L=\bar{Y}_{1} \circ Y_{M_{1}} \circ Q$ for any $L \in\{L \mid$ and the corresponding $Q$. Consequently $\bar{Y}_{1} \circ Y_{M_{1}} \circ Q=\bar{X}_{M_{1}} \circ X_{1} \circ L=\bar{X}_{M_{1}} \circ Y_{1} \circ Q_{1}=\bar{Y}_{1} \circ X_{M_{1}} \circ Q_{1}$. Cancelling $\bar{Y}_{1}$ we get $Y_{M_{1}} \circ Q=X_{M_{1}} \circ Q_{1}$ and finally

$$
\begin{aligned}
& X_{M_{1}} \circ M_{1}=Y_{M_{1}} \circ R, \\
& X_{M_{1}} \circ Q_{1}=Y_{M_{1}} \circ Q,
\end{aligned}
$$

which shows that any $Q_{1}$ in (11) (it depends on $L \in\{L \mid$ ) form a divisor operator couple for $M_{1}$ with any $\left.R \in \mid R\right\}$ so the condition a) of Definition 6 holds. In order to prove the condition c) of it for $\left\{Q_{1}\left|M_{1}\right| R\right\}$ we suppose (12) to be true for some LPDO $R$ and all $Q_{1}$ obtained from (11) with $L \in\left\{L \mid\right.$. Fixing $X_{M_{1}}, Y_{M_{1}}, X_{1}, Y_{1}$ and using the same definition of $\bar{X}_{M_{1}}, \bar{Y}_{M_{1}}: Z_{2}=\bar{Y}_{1} \circ X_{M_{1}}=\bar{X}_{M_{1}} \circ Y_{1}$ we get $\bar{Y}_{1} \circ X_{M_{1}} \circ M_{1}=\bar{X}_{M_{1}} \circ Y_{1} \circ M_{1}=\bar{Y}_{1} \circ Y_{M_{1}} \circ R=\bar{X}_{M_{1}} \circ X_{1} \circ M$, $\bar{Y}_{1} \circ X_{M_{1}} \circ Q_{1}=\bar{Y}_{1} \circ Y_{M_{1}} \circ Q=\bar{X}_{M_{1}} \circ Y_{1} \circ Q_{1}=\bar{X}_{M_{1}} \circ X_{1} \circ L$ so

$$
\begin{aligned}
& \bar{X}_{M_{1}} \circ X_{1} \circ M=\bar{Y}_{1} \circ Y_{M_{1}} \circ R, \\
& \bar{X}_{M_{1}} \circ X_{1} \circ L=\bar{Y}_{1} \circ Y_{M_{1}} \circ Q,
\end{aligned}
$$

that is (9) holds (Lemma 11) for this $R$ and all $L \in\{L \mid$ so $R \in \mid R\}$. We prove the condition b) of Definition 6 in the same way: if (12) holds for some LPDO $Q_{1}$ and all $R \in\{R\}$ $\left(Q, X_{M_{1}}, Y_{M_{1}}\right.$ depend on $R$ ) we take $\left.R=M \in \mid R\right\}$ so (12) becomes

$$
\begin{aligned}
\widetilde{X}_{M_{1}} \circ M_{1} & =\widetilde{Y}_{M_{1}} \circ M, \\
\widetilde{X}_{M_{1}} \circ Q_{1} & =\widetilde{Y}_{M_{1}} \circ \widetilde{Q} .
\end{aligned}
$$

In this case we see from (11) that actually we may set (Lemma 11) $\widetilde{X}_{M_{1}}=Y_{1}, \widetilde{Y}_{M_{1}}=X_{1}, L:=\widetilde{Q}$. So we have 111), (12) with $R \in \mid R\}$ and we shall prove (9) for the constructed $L, Q, M, R$. Again $Z_{2}=\bar{Y}_{1} \circ X_{M_{1}}=\bar{X}_{M_{1}} \circ Y_{1}$, $\bar{X}_{M_{1}} \circ Y_{1} \circ M_{1}=\bar{X}_{M_{1}} \circ X_{1} \circ M=\bar{Y}_{1} \circ X_{M_{1}} \circ M_{1}=\bar{Y}_{1} \circ Y_{M_{1}} \circ R$, $\bar{X}_{M_{1}} \circ X_{1} \circ L=\bar{X}_{M_{1}} \circ Y_{1} \circ Q_{1}=\bar{Y}_{1} \circ X_{M_{1}} \circ Q_{1}=\bar{Y}_{1} \circ Y_{M_{1}} \circ Q$, i.e. we have (13) again or (Lemma 11) we have (9).

Lemma 3 essentially says that the right parts of $\{L|M| R\}$ are internally characterizable as some special left ideals. The same is true for $\{L \mid$. We will call such $\mid R\}$ right divisor ideals or r.d.i. (they are left ideals of the ring of LPDO :-) and $\{L \mid$ - left divisor ideals or 1.d.i. (they are right ideals). Any principal left ideal is a r.d.i.: $\left.\left|R_{0}\right\rangle=\mid R_{0}\right\}$ since we obviously have $\left\{1\left|R_{0}\right| R_{0}\right\}$, that is (9) with $R=P \circ R_{0}, M=R_{0}$, $Y=1, X=P, Q=P \circ L,(P, L$ being arbitrary LPDO $)$.

A divisor ideal couple $\{L|M| R\}$ is called trivial if either $\{L \mid=\langle M|$ (then $\mid R\}=0=|1\rangle)$ or $\mid R\}=|M\rangle,\{L \mid=0$.

On the other hand as we will see in the next section there are divisor ideals which are not principal and not every (left) ideal is r.d.i.

Let us now prove that the set of r.d.i. with the natural ordering $\left.\left(\mid R_{1}\right\} \geq \mid R_{2}\right\}$ iff $\left.\left.\left.\mid R_{1}\right\} \subset \mid R_{2}\right\}\right)$ forms a lattice. Namely for two r.d.i. $\left.\left.\mid R_{1}\right\}, \mid R_{2}\right\}$ we take their intersection as their $\left.\left.\left.\left.\operatorname{lLCM}: \operatorname{lLCM}\left(\mid R_{1}\right\}, \mid R_{2}\right\}\right):=\sup \left(\mid R_{1}\right\}, \mid R_{2}\right\}\right):=$ $\left.\left.\left.\left.\mid R_{1}\right\}+\mid R_{2}\right\}:=\mid R_{1}\right\} \bigcap \mid R_{2}\right\}$. Then for $\left.\left.M \in \mid R_{1}\right\} \bigcap \mid R_{2}\right\}$ we find two corresponding l.d.i. (Lemma 3): $\left\{L_{1}|M| R_{1}\right\}$, $\left\{L_{2}|M| R_{2}\right\}$. Now let us take all LPDO $L$ such that (9) holds for every $\left.\left.R \in \mid R_{1}\right\} \bigcap \mid R_{2}\right\}$. The right ideal $\{L \mid$ of such operators forms the divisor ideal couple with $\left.\left.\mid R\}=\mid R_{1}\right\} \bigcap \mid R_{2}\right\}$ for $M$ since a) and b) in Definition 6 hold automatically and $\left\{L \mid \supset\left\{L_{1} \mid \bigcup\left\{L_{2} \mid\right.\right.\right.$ so every operator $R$ such that (9) holds for all $L \in\{L \mid$ forms a divisor operator couple with 
all $L \in\left\{L_{1} \mid\right.$ and $L \in\left\{L_{2} \mid\right.$ so $\left.\left.R \in \mid R_{1}\right\} \bigcap \mid R_{2}\right\}$ and the condition c) holds. As we will see in the next section the constructed $\{L \mid$ is in general greater than the set of all sums of elements of $\left\{L_{1} \mid\right.$ and $\left\{L_{2} \mid\right.$. Obviously this $\{L \mid$ plays the role of $\operatorname{lGCD}\left(\left\{L_{1} \mid,\left\{L_{2} \mid\right) \equiv\left\{L_{1} \mid \cdot\left\{L_{2} \mid\right.\right.\right.\right.$ in the poset of all l.d.i. The determination of $\left.\left.\mid R\}=\operatorname{rGCD}\left(\mid R_{1}\right\}, \mid R_{2}\right\}\right):=$ $\left.\left.\left.\left.\inf \left(\mid R_{1}\right\}, \mid R_{2}\right\}\right):=\mid R_{1}\right\} \cdot \mid R_{2}\right\}$ is obtained in the same way: we now take $\left\{L \mid=\operatorname{rLCM}\left(\left\{L_{1} \mid,\left\{L_{2} \mid\right):=\left\{L_{1} \mid \bigcap\left\{L_{2} \mid\right.\right.\right.\right.\right.$ and the corresponding $\left.\left.\mid R\} \supset \mid R_{1}\right\} \bigcup \mid R_{2}\right\}$ is defined using (9). So our lattice of r.d.i. does not form a sublattice of the lattice of all right ideals of LPDO, it changes inf; such subsets are called "meet-sublattices".

\section{Coordinatization of divisor ideals}

Any (non-commutative) ring $R$ satisfying the so called Ore condition (absence of zero divisors and existence of at least one common multiple for every two non-zero elements) may be imbedded into a skew field (non-commutative ring with division) built with formal quotients $L^{-1} \circ M=\bar{M} \circ \bar{L}^{-1}$, $L, M, \bar{L}, \bar{M} \in R(20])$. Let us take $R=\mathbf{Q}(x, y)\left[D_{x}\right]$ and the corresponding skew field $\mathbf{Q}\left(x, y, D_{x}\right)$. We can form a $\operatorname{ring} \mathbf{Q}\left(x, y, D_{x}\right)\left[D_{y}\right]$ of operators of the type $L=a_{0} D_{y}^{n}+$ $a_{1} D_{y}^{n-1}+\ldots+a_{n}, a_{i}=\left(L_{i}\right)^{-1} \circ M_{i} \in \mathbf{Q}\left(x, y, D_{x}\right)$ if we define the corresponding $D_{y}$-differentiation for the coefficients:

$\partial\left(\left(L_{i}\right)^{-1} \circ M_{i}\right) / \partial y=\left(L_{i}\right)^{-1} \circ \partial\left(M_{i}\right) / \partial y-\left(L_{i}\right)^{-1} \circ L_{i}^{\prime} \circ\left(L_{i}\right)^{-1}$ with $\partial\left(M_{i}\right) / \partial y=\partial\left(m_{0}(x, y) D_{x}^{k}+\ldots+m_{k}(x, y)\right) / \partial y=$ $\left(\partial m_{0}(x, y) / \partial y\right) D_{x}^{k}+\ldots+\partial m_{k}(x, y) / \partial y ; L_{i}^{\prime} \in \mathbf{Q}(x, y)\left[D_{x}\right]$ is defined via $D_{y} \circ L_{i}=L_{i} \circ D_{y}+L_{i}^{\prime} \Rightarrow L_{i}^{-1} \circ D_{y}=D_{y} \circ$ $L_{i}^{-1}+L_{i}^{-1} \circ L_{i}^{\prime} \circ L_{i}^{-1}$. As explained in 19$]$ the basic facts of the Loewy-Ore theory (namely the existence of the Euclid division algorithm, GCDs, LCMs) hold also for operators with coefficients in differential skew fields. Any (left) ideal is again a principal ideal, they form a modular lattice.

We have the natural projections $\mathcal{P}_{x}: \mathbf{Q}(x, y)\left[D_{x}, D_{y}\right] \rightarrow$ $\mathbf{Q}\left(x, y, D_{y}\right)\left[D_{x}\right]$ and $\mathcal{P}_{y}: \mathbf{Q}(x, y)\left[D_{x}, D_{y}\right] \rightarrow \mathbf{Q}\left(x, y, D_{x}\right)\left[D_{y}\right]$. For any left ideal $I \subset \mathbf{Q}(x, y)\left[D_{x}, D_{y}\right]$ we obtain two principal ideals $\mathcal{P}_{x}(I)=\left|I_{x}\right\rangle, \mathcal{P}_{y}(I)=\left|I_{y}\right\rangle$, their generators $I_{x}$, $I_{y}$ are called coordinates of $I, I_{x} \in \mathbf{Q}\left(x, y, D_{y}\right)\left[D_{x}\right], I_{y} \in$ $\mathbf{Q}\left(x, y, D_{x}\right)\left[D_{y}\right]$, we also normalize them $\operatorname{lcof}_{D_{x}}\left(I_{x}\right)=1$, $\operatorname{lcof}_{D_{y}}\left(I_{y}\right)=1$. Strictly speaking $\mathcal{P}_{x}(I)$ and $\mathcal{P}_{y}(I)$ are not ideals in the respective rings; in order to make them ideals we need to multiply all their element by various $L^{-1}$, $L \in \mathbf{Q}(x, y)\left[D_{x}\right]$ (resp. $\left.L \in \mathbf{Q}(x, y)\left[D_{y}\right]\right)$. This will be implicitly done hereafter.

First we remark that if we have $\{L|M| R\}$ then for the coordinates

$$
\mathcal{P}_{x}(M)=L_{x} \circ R_{x}
$$

which gives a heuristic foundation for the definitions of the previous section. Also obviously $\left.\left.\mathcal{P}_{x}\left(\operatorname{lLCM}\left(\mid R_{1}\right\}, \mid R_{2}\right\}\right)\right)=$ $\left.\left.\operatorname{lLCM}\left(\mathcal{P}_{x}\left(\mid R_{1}\right\}\right), \mathcal{P}_{x}\left(\mid R_{2}\right\}\right)\right)$ which in turn gives

$\mathcal{P}_{x}\left(\operatorname{lGCD}\left(\left\{L_{1} \mid,\left\{L_{2} \mid\right)\right)=\operatorname{lGCD}\left(\mathcal{P}_{x}\left(\left\{L_{1} \mid\right), \mathcal{P}_{x}\left(\left\{L_{2} \mid\right)\right)\right.\right.\right.\right.$ due to (14). Symmetrically $\left.\left.\left.\left.\mathcal{P}_{x}\left(\operatorname{rGCD}\left(\mid R_{1}\right\}, \mid R_{2}\right\}\right)\right)=\operatorname{rGCD}\left(\mathcal{P}_{x}\left(\mid R_{1}\right\}\right), \mathcal{P}_{x}\left(\mid R_{2}\right\}\right)\right)$, $\mathcal{P}_{x}\left(\operatorname{rLCM}\left(\left\{L_{1} \mid,\left\{L_{2} \mid\right)\right)=\operatorname{rLCM}\left(\mathcal{P}_{x}\left(\left\{L_{1} \mid\right), \mathcal{P}_{x}\left(\left\{L_{2} \mid\right)\right)\right.\right.\right.\right.$.

The following lemma plays the key role in the subsequent proofs.

Lemma 4 If a r.d.i. $\mid R\} \subset \mathbf{Q}(x, y)\left[D_{x}, D_{y}\right]$ contains two elements $A \circ P, B \circ P$ such that $A \in \mathbf{Q}(x, y)\left[D_{x}\right], B \in$ $\mathbf{Q}(x, y)\left[D_{y}\right]$ then $\left.P \in \mid R\right\}$.

Proof. Using (10) we obtain $A \circ P \circ \bar{X}=Q \circ \bar{Y}, B \circ P \circ$ $\bar{X}=S \circ \bar{Y}$ for some $S, Q$. Then $P \circ \bar{X}=A^{-1} \circ Q \circ \bar{Y} \in$

$$
\begin{gathered}
\mathbf{Q}\left(x, y, D_{x}\right)\left[D_{y}\right], B \circ P \circ \bar{X}=B \circ A^{-1} \circ Q \circ \bar{Y}=S \circ \bar{Y} \Rightarrow \\
B \circ A^{-1} \circ Q=S \in \mathbf{Q}(x, y)\left[D_{x}, D_{y}\right],
\end{gathered}
$$

$B \in \mathbf{Q}(x, y)\left[D_{x}\right]$. We will prove that in such circumstances $Q$ is divisible by $A$ : $Q=A \circ \bar{Q}, \bar{Q} \in \mathbf{Q}(x, y)\left[D_{x}, D_{y}\right]$. Without loss of generality we may suppose $\operatorname{lcof}(B)=1$ so (15) reads

$$
\underbrace{\left(D_{y}^{m}+b_{1}(x, y) D_{y}^{m-1}+\ldots+b_{m}(x, y)\right)}_{B} \circ
$$

$$
\underbrace{\left(C_{0} D_{y}^{n}+C_{1} D_{y}^{n-1}+\ldots+C_{n}\right)}_{A^{-1} \circ Q}=S,
$$

with $C_{i} \in \mathbf{Q}\left(x, y, D_{y}\right)$. The leading coefficient of the l.h.s. (in $\mathbf{Q}\left(x, y, D_{x}\right)\left[D_{y}\right]$ ) of (16) is $C_{0}$ so since $S \in \mathbf{Q}(x, y)\left[D_{x}, D_{y}\right]$, $C_{0} \in \mathbf{Q}(x, y)\left[D_{x}\right]$. Then the coefficient of $D_{y}^{m+n-1}$ in (16) will be $C_{1}+\partial C_{0} / \partial y+b_{1} C_{0} \in \mathbf{Q}(x, y)\left[D_{x}\right]$ so also $C_{1} \in$ $\mathbf{Q}(x, y)\left[D_{x}\right]$. Using induction we get $C_{i} \in \mathbf{Q}(x, y)\left[D_{x}\right]$ so $A^{-1} \circ Q=\bar{Q} \in \mathbf{Q}(x, y)\left[D_{x}, D_{y}\right]$. This gives us the possibility to cancel $A$ in $A \circ \bar{X}=Q \circ \bar{Y}$ obtaining $\bar{X}=\bar{Q} \circ \bar{Y}$. Then the operator $P \in \mid R\}$ since $P \circ \bar{X}=(P \circ \bar{Q}) \circ \bar{Y}$ in $(10)$.

Remark. Actually we used only the fact $\operatorname{lcof}_{D_{y}}(B)=1$.

Corollary 1 If a r.d.i. $\mid R\}$ contains two elements $A, B$, $A \in \mathbf{Q}(x, y)\left[D_{x}\right], B \in \mathbf{Q}(x, y)\left[D_{y}\right]$ then $\left.\mid R\right\}$ is trivial, $\left.\mid R\right\}=$ $0=|1\rangle=\mathbf{Q}(x, y)\left[D_{x}, D_{y}\right]$.

This Corollary explains why r.d.i. are "codimension 1" ideals: if we will take a "codimension 2 " left ideal generated by $D_{x}^{n}, D_{y}^{m}$, (solutions of the corresponding system $D_{x}^{n} f=0$, $D_{y}^{m} f=0$ are functions of 2 variables parameterized by several constants and not by functions of 1 variable), it is contained only in the trivial r.d.i. $\mid 1\}$. The set of divisor ideals is a bit larger than the set of principal ideals (which are obviously "codimension 1" ideals - solutions of the corresponding system of 1 equation are parameterized by functions of 1 variable).

Theorem 5 Two r.d.i. $\left.\left.\mid R_{1}\right\}, \mid R_{2}\right\}$, coincide iff their coordinates coincide i.e. iff $\left.\left.\left.\mathcal{P}_{x}\left(\mid R_{1}\right\}\right)=\mathcal{P}_{x}\left(\mid R_{2}\right\}\right), \mathcal{P}_{y}\left(\mid R_{1}\right\}\right)=$ $\left.\mathcal{P}_{y}\left(\mid R_{2}\right\}\right)$.

Proof. Let some $\left.R \in \mid R_{1}\right\}$. Since $\left.\left.\mathcal{P}_{x}\left(\mid R_{1}\right\}\right)=\mathcal{P}_{x}\left(\mid R_{2}\right\}\right)$ we may find $\left.\left.\bar{R} \in \mid R_{2}\right\}\right)$, such that $C \circ R=\bar{R}, C=\left(C_{1}\right)^{-1} C_{2} \in$ $\mathbf{Q}\left(x, y, D_{y}\right)$. Multiplying with $C_{1}$ we get $C_{2} \circ R=C_{1} \circ \bar{R}=$ $\left.\widetilde{R} \in \mid R_{2}\right\}$. Hence for some $C_{2} \in \mathbf{Q}(x, y)\left[D_{y}\right]$ we have $C_{2} \circ R \in$ $\left.\mid R_{2}\right\}$. Analogous consideration w.r.t. $\mathcal{P}_{y}$ give $\left.K_{2} \circ R \in \mid R_{2}\right\}$ for $K_{2} \in \mathbf{Q}(x, y)\left[D_{x}\right]$, thus $\left.R \in \mid R_{2}\right\}$ (Lemma i) hence $\left.\left.\mid R_{1}\right\} \subset \mid R_{2}\right\}$. Symmetrically $\left.\left.\mid R_{2}\right\} \subset \mid R_{1}\right\}$.

Remark. This is obviously not true for arbitrary ideals: the ideal generated by $D_{x}, D_{y}$ have the same projections as the trivial $|1\rangle$.

Corollary 2 The lattice of r.d.i. (l.d.i.) is modular.

Proof. Since the modular identity (3) holds for projections due to modularity of the lattice of the (principal) left ideals of $\mathbf{Q}\left(x, y, D_{x}\right)\left[D_{y}\right], \mathbf{Q}\left(x, y, D_{y}\right)\left[D_{x}\right]$, and GCD, LCM are preserved after projections, we conclude $\mathcal{P}_{x}((A \cdot C+B) \cdot C)=$ $\mathcal{P}_{x}(A \cdot C+B \cdot C), \mathcal{P}_{y}((A \cdot C+B) \cdot C)=\mathcal{P}_{y}(A \cdot C+B \cdot C)$, so $(A \cdot C+B) \cdot C=A \cdot C+B \cdot C$. 
Corollary 3 For any r.d.i. $\mid R\}$ the length $k+1$ of a chain of r.d.i. $\left.\left.\mid R\}>\mid R_{1}\right\}>\ldots>\mid R_{k}\right\}>0$ is limited: $k+1 \leq$ $\left.\left.\operatorname{ord}_{D_{x}}(\mid R\}_{x}\right)+\operatorname{ord}_{D_{y}}(\mid R\}_{y}\right)$.

Proof. Since $\left.\left.\mathcal{P}_{x}\left(\mid R_{i}\right\}\right), \mathcal{P}_{y}\left(\mid R_{i}\right\}\right)$ give chains of divisors of $\left.\left.\mathcal{P}_{x}(\mid R\}\right), \mathcal{P}_{y}(\mid R\}\right)$ in $\mathbf{Q}\left(x, y, D_{y}\right)\left[D_{x}\right]\left(\right.$ resp. $\left.\mathbf{Q}\left(x, y, D_{x}\right)\left[D_{y}\right]\right)$, the adjacent elements of $\mathcal{P}_{x}$-projected chain may differ only in $\left.\operatorname{ord}_{D_{x}}(\mid R\}_{x}\right)$ places (resp. in ord $\left.D_{D_{y}}(\mid R\}_{y}\right)$ places).

Analogous result is true for (direct) sup-sums of r.d.i.

Thus Theorems 14 are applicable to the constructed lattice of r.d.i. (l.d.i.) of a given LPDO.

Proposition 2 Any first order LPDO R is irreducible (i.e. it has no nontrivial divisor ideal couples).

Proof. Let $R=r_{1}(x, y) D_{x}+r_{2}(x, y) D_{y}+r_{3}(x, y)$ but nevertheless we have some r.d.i. $\left.\left.\left.\mid R_{1}\right\}, \mid R\right\}>\mid R_{1}\right\}>0$. Necessarily $\left.\left.\operatorname{ord}_{D_{x}}\left(\mid R_{1}\right\}_{x}\right)=1, \operatorname{ord}_{D_{y}}\left(\mid R_{1}\right\}_{y}\right)=0$ (up to transposition $x \leftrightarrow y$ ), so there exist $\left.R_{1,1}=C_{0} \circ D_{x}+C_{1} \in \mid R_{1}\right\}$, $\left.R_{1,2}=K_{1} \in \mid R_{1}\right\}, C_{i} \in \mathbf{Q}(x, y)\left[D_{y}\right], K_{1} \in \mathbf{Q}(x, y)\left[D_{x}\right]$. Since $\left.\left.\mid R\} \subset \mid R_{1}\right\}, R_{1,3}=R_{1,1}-\frac{C_{0}}{r_{2}(x, y)} R=K_{2} \in \mid R_{1}\right\}$, $K_{2} \in \mathbf{Q}(x, y)\left[D_{y}\right]$. Due to Corollary 1 of Lemma 4 we have $\left.\mid R_{1}\right\}=0=\mathbf{Q}(x, y)\left[D_{x}, D_{y}\right]$.

Proposition 3 Any LODO $M \in \mathbf{Q}(x)\left[D_{x}\right]$ irreducible in this ring is irreducible as an element of $\mathbf{Q}(x, y)\left[D_{x}, D_{y}\right]$.

Proof. Suppose we have a nontrivial divisor ideal couple $\{L|M| R\}$ so for some $L \in\{L|, R \in| R\}$ we have (10). Since $M \in\{L|, M \in| R\}$ we may suppose (subtracting a suitable multiple of $M)$ that $\operatorname{ord}_{D_{x}}(L)=\operatorname{ord}\left(\mathcal{P}_{x}(L)\right)<$ $\operatorname{ord}_{D_{x}}(M)=m, \operatorname{ord}_{D_{x}}(R)<m$. Forming the quotient skew field $\mathbf{Q}\left(x, y, D_{x}, D_{y}\right)$ we can write in this skew field $X=Y \circ Q \circ L^{-1} \Rightarrow X \circ M=Y \circ Q \circ L^{-1} \circ M=Y \circ R \Rightarrow$ $Q \circ L^{-1} \circ M=R$. If we will find $\operatorname{lLCM}\left(\mathcal{P}_{x}(Q), \mathcal{P}_{x}(L)\right)=$ $Z=\bar{Q} \circ L=\bar{L} \circ Q_{x} \in \mathbf{Q}\left(x, y, D_{y}\right)\left[D_{x}\right]$ then $\operatorname{ord}_{D_{x}}(\bar{L}) \leq$ $\operatorname{ord}_{D_{x}}(L)<m$. For some $C \in \mathbf{Q}(x, y)\left[D_{y}\right]$ we get $C$ 。 $\bar{Q}=\widetilde{Q} \in \mathbf{Q}(x, y)\left[D_{x}, D_{y}\right], C \circ \bar{L}=\widetilde{L} \in \mathbf{Q}(x, y)\left[D_{x}, D_{y}\right]$, $\operatorname{ord}_{D_{x}}(\widetilde{L})=\operatorname{ord}_{D_{x}}(\bar{L})<m$. Then $\widetilde{L} \circ Q \circ L^{-1} \circ M=\widetilde{L} \circ R$ $\Leftrightarrow \widetilde{Q} \circ L \circ L^{-1} \circ M=\widetilde{Q} \circ M=\widetilde{L} \circ R$. Let us take $\operatorname{lcof}_{D_{y}}(\widetilde{L} \circ$ $R):=\operatorname{lcof}\left(\mathcal{P}_{y}(\widetilde{L} \circ R)\right)=\operatorname{lcof}_{D_{y}}(\widetilde{L}) \cdot \operatorname{lcof}_{D_{y}}(R)=C_{\widetilde{L}} \circ C_{R} \in$ $\mathbf{Q}(x, y)\left[D_{x}\right], \operatorname{ord}\left(C_{\widetilde{L}}\right)<m, \operatorname{ord}\left(C_{R}\right)<m, \operatorname{lcof}_{D_{y}}(\widetilde{Q} \circ M)=$ $\operatorname{lcof}_{D_{y}}(\widetilde{Q}) \circ M=C_{\widetilde{Q}} \circ M, \operatorname{ord}(M)=m$. Finally we have $C_{\widetilde{L}} \circ C_{R}=C_{\widetilde{Q}} \circ M$ in $\mathbf{Q}(x, y)\left[D_{x}\right]$ which contradicts to the fact that $M$ is irreducible, $\operatorname{ord}(M)=m$ but the orders of the factors in the l.h.s. are $<m$.

Finally we are able to explain the mystery of the example of E.Landau (1): due to Proposition 2 the chain $\mid Q \circ Q \circ$ $P\}>\mid Q \circ P\}>\mid P\}>0$ is maximal, so the only place where our generalized divisors appear is the interval between $\mid R \circ Q\}$ and $\mid Q\}$ in $\mid R \circ Q\}>\mid Q\}>0$, namely we insert here the $I=\operatorname{lLCM}(\mid Q\}, \mid P\})$. In terms of projections $\mathcal{P}_{x}(I)$ is generated by second order (w.r.t. $D_{x}$ ) operator $L_{31}=$ $\left(x^{2} D_{y}^{2}+x D_{x} D_{y}-(x+1) D_{y}-D_{x}\right) \circ Q$ while $\mathcal{P}_{x}(R \circ Q)$ is a third-order operator. So the operator $R$ is reducible in our sense (and reducible in $\mathbf{Q}\left(x, y, D_{y}\right)\left[D_{x}\right]$ ) and it has a nontrivial "generalized common (right) divisor" with $x^{2} D_{y}^{2}+$ $x D_{x} D_{y}-(x+1) D_{y}-D_{x}$ (Definition 5).

\section{Darboux integrability of nonlinear PDEs and factoriza- tion of linearized equations}

In the XIX-th century vast interest in finding exact solutions to partial differential equations resulted in the development of methods of Lagrange, Monge, Boole and Ampere.
G. Darboux [6] generalized the method of Monge (known as the method of intermediate integrals) to obtain the most powerful method for exact integration of partial differential equations known in the last century.

Recently in a series of papers [1, 14, 26, the Darboux method was cast into a more precise and efficient (although not completely algorithmic) form. For the case of a single second-order nonlinear PDE of the form

$$
u_{x y}=f\left(x, y, u, u_{x}, u_{y}\right)
$$

their idea consisted in linearization of (17): using substitution $u(x, y) \rightarrow u(x, y)+\epsilon v(x, y)$ and cancelling terms with $\epsilon^{n}, n>1$, we obtain a LPDE

$$
v_{x y}=A v_{x}+B v_{y}+C v
$$

with coefficients depending on $x, y, u, u_{x}, u_{y}$. Equations of the type (18) were studied by Laplace, who invented a method of transformation (called sometimes the Laplace cascade method) of (18). First af all we remark that for the corresponding LPDOL $=D_{x} \circ D_{y}-A \cdot D_{x}-B \cdot D_{y}-C$,

$$
L=\left(D_{x}-B\right) \circ\left(D_{y}-A\right)+H=\left(D_{y}-A\right) \circ\left(D_{x}-B\right)+K,
$$

where $H=D_{x} A-A B-C \cdot K=D_{y} B-A B-C$, are called the Laplace invariants of 18 . So if eihter $H \equiv 0$ or $K \equiv 0$ our second-order LPDO $L$ factors in the "usual" sense and the solutions of (18) may be found via quadratures. If both $H, K$ vanish, $L$ is a ILCM of two first-order LPDO. If both $H, K$ do not vanish, one can apply the two Laplace transformations: $L \rightarrow L_{1}, L \rightarrow L_{-1}$, usung the substitutions

$$
v_{1}=\left(D_{y}-A\right) v, \quad v_{-1}=\left(D_{x}-B\right) v .
$$

These (invertible) transformations give two new second order LPDO $L_{1}, L_{-1}$ of the same form with different coefficients iff $H \neq 0$ (resp. $K \neq 0$ ). In the generic case one obtains two infinite sequences

$$
\begin{gathered}
L \rightarrow L_{1} \rightarrow L_{2} \rightarrow \cdots, \\
L \rightarrow L_{-1} \rightarrow L_{-2} \rightarrow \cdots .
\end{gathered}
$$

If one of these sequences is finite (i.e. the corresponding Laplace invariant vanishes and the Laplace transform can not be applied once more) then the final LPDO $L_{i}$ is trivially factorable.

One shall certainly take into consideration the original equation (17) performing all the computations of the Laplace invariants and Laplace transforms (which allows us to express all the mixed derivatives of $u$ via $x, y, u$ and the nonmixed $\left.u_{x \cdots x}, u_{y \cdots y}\right)$.

Theorem 6 (䚾, 14, 26) A second order, scalar, hyperbolic partial differential equation (17) is Darboux integrable if and only if both Laplace sequences are finite.

In 11, 14, 26] this method was also generalized for the case of a general second-order nonlinear PDE

$$
F\left(x, y, u, u_{x}, u_{y}, u_{x x}, u_{x y}, u_{y y}\right)=0
$$

What can be said about factorizability of the operator $L$ in our generalized sense?

Theorem $7 L=D_{x} \circ D_{y}-a(x, y) D_{x}-b(x, y) D_{y}-c(x, y)$ has a nontrivial generalized right divisor ideal iff one of the Laplace sequences is finite. 
Theorem $8 L=D_{x} \circ D_{y}-a(x, y) D_{x}-b(x, y) D_{y}-c(x, y)$ is a lLCM of two generalized right divisor ideals iff both Laplace sequences are finite.

The detailed proofs will be given elsewhere.

In fact these theorems demonstrate again that the "generalized" factorization introduced here enjoys the necessary natural properties: it is invariant w.r.t. the differential substitutions (20) (which destroy the "trivial" factorizations $L=\left(D_{x}-B\right) \circ\left(D_{y}-A\right)$ or $\left.L=\left(D_{y}-A\right) \circ\left(D_{x}-B\right)\right)$.

\section{Conclusion}

An obvious and important generalization of our definition of reducibility (or sup-decomposition) of single LPDO would be a proper definition of decomposition of systems of LPDO (6). Actually a formal generalization should be formulated inductively; for example if a system of 2 equations has indecomposable first equation $L_{1} f=0$ then we may try to find (generalized) divisor operator couples for the second LPDO $L_{2}$ (i.e. the second equation $L_{2} f=0$ ) forming for $M=L_{2}$ equations (9) modulo the left principal ideal generated by $L_{1}$. The problem of zero-divisors (actually any LPDO is zero divisor now since it always has a multiple which belongs to the ideal $\left|L_{1}\right\rangle$ ) is (apparently) solved using the fact that for non-decomposable $L_{1}$ there are no "LCM-zero divisors", i.e. if $M \notin\left|L_{1}\right\rangle, R \notin\left|L_{1}\right\rangle$ then some their right c.m. $M \circ X=R \circ Y \notin\left|L_{1}\right\rangle$. In fact our proofs rely only on absence of "LCM-zero divisors". Certainly this approach deserves further thorough study in another publication. Especially interesting is the possibility to apply such a generalization to the commutative case (factorized Gröbner bases).

It would be interesting to compare our definitions of decomposition of ideals with the known results on decomposition of ideals in non-commutative rings with the Ore condition (existence of at least one common multiples for every two elements) [12].

A more challenging generalization is required for treatment of overdetermined linear partial differential systems with several unknown functions $f_{k}$.

The algebraic nature of the set of operators $\{Q\}$ in (9), (10) also is of interest: we can multiply $Q$ on the left and on the right with arbitrary LPDO, but addition of different $Q_{i}$ is doubly "stratified": only $Q_{i}$ which belong to a fixed $R$ or a fixed $L$ may be added. As we have seen in the proof of Lemma 3 each "stratum" of $\{Q\}$ is actually some $\mid R\}$ (resp. $\{L \mid)$.

An algorithm of computation of divisor ideals for a given LPDO would be of big practical interest. As we have explained in Introduction an algorithm for sup-decompositions is much more important for applications (Theorems 2, 3). One possible approach for algorithmization of sup-decompositions may mimic the methods of [24]. For this purpose we have to generalize the eigenring algorithm of [24] to the case of skew differential fields of coefficients $\overline{\mathbf{Q}}\left(x, y, D_{x}\right)$ with greater (not algebraically closed) constant subfield $\overline{\mathbf{Q}}\left(D_{x}\right)$. Another approach to reducibility testing may rely on possible generalization of estimates of complexity of coefficients of factors given in [8] for commutative coefficient fields to the case of the ring $\mathbf{Q}\left(x, y, D_{x}\right)\left[D_{y}\right]$. These difficult problems are far beyond the scope of this short communication.

The theorems proved in section 4 may provide a basis for algorithmic checking of Darboux integrability of nonlinear PDEs (provided a suitable factorisation algorithm for corresponding linearized equations with coefficients depending on solutions of another PDEs will be found). Also we may conjecture that a generalization of the Darboux integrability method to PDEs of higher order with arbitrary number of independent variables may be given: such integrability should be related to representation of the corresponding linearized LPDO as a ILCM of "first-order" generalised divisor ideals.

\section{Acknowledgements}

The author wishes to express his special gratitude to Prof. F. Schwarz (GMD, St. Augustin, Germany) who communicated the informative and stimulating example (1) and provided a copy of [5]. Many useful discussions about lattice theory with Prof. B.V.Yakovlev and Dr. A.M.Kut'in (Krasnoyarsk) are thankfully acknowledged.

\section{References}

[1] Anderson, I.M., And Kamran, N. The Variational Bicomplex for Second Order Scalar Partial Differential Equations in the Plane. CRM technical report, Utah State University, September 1994.

[2] BEKE, E. Die Irreducibilität der homogenen linearen Differentialgleichungen. Math. Annalen 45 (1894), pp. 278-300.

[3] Birknoff, G. Lattice theory. 1967, AMS, Providence, R.I.

[4] BJÖRK, J. E. Rings of differential operators. 1979, North-Holland.

[5] BlumberG, H. Über algebraische Eigenschaften von linearen homogenen Differentialausdrücken. Diss., Göttingen. 1912.

[6] Darboux, G. Sur les équations aux dérivées partielles du second ordre. Ann. Ecole Normale Sup., t. VII (1870), pp. 163-173.

[7] Grätzer, G. General lattice theory. 1978, AkademieVerlag, Berlin.

[8] Grigor'ev, D. Yu. Complexity of factoring and calculating the GCD of linear ordinary differential operators, J. Symbolic Computation 10 (1990), pp. 7-37.

[9] Grigor'ev, D. Yu. Complexity of computing the genus of a system of exterior differential equations. Soviet Math. - Doklady 39 (1989), No 3, pp. 432-436.

[10] Ireland, K., And Rosen, M. A Classical Introduction to Modern Number Theory. 1982, Springer-Verlag.

[11] Jacobson, N. Lectures in abstract algebra. v.1. 1951.

[12] Jacobson, N. The theory of rings. 1943. AMS Math. Surveys, N.Y.

[13] JAnet, M. Leçons sur les systèmes d'equations aux derivées partielles. 1929, Gautier-Villard.

[14] Juras, M. Generalized Laplace invariants and classical integration methods for second order scalar hyperbolic partial differential equations in the plane. Proc. Conf. DIFFERENTIAL GEOMETRY AND APPLICATIONS, Aug. 28 - Sept. 1, 1995, Brno, Czech Republic Masaryk University, Brno 1996, pp. 275-284. 
[15] Landau, E. Ein Satz über die Zerlegung homogener linearer Differentialausdrücke in irreducible Factoren. J. für die reine und angewandte Math. 124 (1901/1902), pp. 115-120.

[16] Loewy, A. Über reduzible lineare homogene Differentialgleichungen. Math. Annalen 56 (1903), pp. 549-584.

[17] LoEwy, A. Über vollstandig reduzible lineare homogene Differentialgleichungen. Math. Annalen 62 (1906), pp. 89-117.

[18] Olver, P. J. Applications of Lie groups to differential equations. 1986, Springer-Verlag.

[19] Ore, O. Theory of non-commutative polynomials. Annals of Mathematics 34 (1933), pp. 480-508.

[20] Ore, O. Linear equations in non-commutative fields. Annals of Mathematics 32 (1931), pp. 463-477.

[21] REID, G. J. Algorithms for reducing a system of PDEs to standard form, determining the dimension of its solution space and calculating its Taylor series solutions. Euro. J. Appl. Mech. 2 (1991), p. 293-318.

[22] Riquier, C. Les systèmes d'equations aux derivées partielles. 1910, Gautier-Villard.

[23] Singer, M. F., And Ulmer, F. Galois groups of second and third order linear differential equations. Journal of Symbolic Computation 16 (1993), pp. 9-36.

[24] Singer, M. F. Testing reducibility of linear differential operators: a group theoretic perspective. Applicable Algebra in Engineering, Communication and Computing 7 (1996), pp. 77-104.

[25] Schwarz, F. The Riquier-Janet theory and its applications to nonlinear evolution equations. Physica D, 11 (1984), p. 243-351.

[26] Sokolov, V.V., And Zhiber, A.V. On the Darboux integrable hyperbolic equations. Physics Letters A 208 (1995), pp. 303-308.

[27] Tsarev, S. P. An algorithm for complete enumeration of all factorizations of a linear ordinary differential operator. Proceedings of ISSAC'96 (1996), ACM Press, pp. 226-231. 